\title{
When the ringing in the ears gets unbearable: Illness representations, self-instructions and adjustment to tinnitus
}

\author{
Manja Vollmann ${ }^{\mathrm{a}, *}$, Natallia Kalkouskaya ${ }^{\mathrm{a}}$, Berthold Langguth ${ }^{\mathrm{b}}$, Margreet Scharloo ${ }^{\mathrm{c}}$ \\ a University of Konstanz, Germany \\ ${ }^{\mathrm{b}}$ Department of Psychiatry and Psychotherapy, University of Regensburg, Germany \\ ${ }^{c}$ Leiden University Medical Center, The Netherlands
}

\section{Keywords:}

Tinnitus

Illness representations

Self-instructions

Adjustment

Coping

Common Sense Model (CSM) of self-regulation

\begin{abstract}
A B S T R A C T
Objective: Chronic tinnitus can severely impair a person's quality of life. The degree of impairment, however, is not closely related to tinnitus loudness. Applying the common sense model (CSM) of self regulation of health and illness, this study investigated to what extent psychological factors, i.e. illness representations and positive/negative self instructions, are associated with the degree of tinnitus related complaints. Methods: In this cross sectional study, 118 patients diagnosed with chronic tinnitus filled in questionnaires assessing illness representations (IPQ R), positive and negative self instructions (TRSS), and tinnitus related complaints (TQ).

Results: The regression analysis yielded a number of significant associations between illness representations and tinnitus related complaints, particularly for the IPQ R dimensions identity, consequences, coherence, and emotional representations. With regard to self instructions and tinnitus related complaints, significant effects were found only for negative self instructions. Moreover, multiple mediation analyses revealed that the effects of consequences and emotional representations on tinnitus related complaints were (partially) due to the use of negative self instructions.

Conclusion: Psychological factors are strongly related to the extent of tinnitus related complaints. The find ings provide an indication of which aspects should be targeted in psychological and psychotherapeutic tinni tus treatment.
\end{abstract}

\section{Introduction}

Tinnitus, defined as the perception of an internal sound in the ab sence of an external stimulus [1], is a widespread phenomenon in the general population [2]. Chronic tinnitus can severely impair a per son's quality of life as it causes a number of psychological and somatic complaints [3 6]. For about $1020 \%$ of affected people, tinnitus be comes an impairing condition. The degree of impairment, however, does not appear to be closely related to objective aspects of the tinni tus, such as its loudness $[7,8]$. This suggests that adjustment to tinni tus is likely to be influenced by psychological factors.

The Common Sense Model (CSM) of self regulation of health and illness [9] addresses how psychological factors, i.e. illness representa tions and coping responses, may influence adjustment to medical conditions. The CSM proposes that individuals' mental representa tions of an illness determine their coping responses which, in turn, have an impact on their illness adjustment. There is growing cross sectional and prospective evidence for the usefulness of the CSM in predicting illness outcomes in a wide range of illnesses [10 12].

Previous studies have examined the impact of illness representa tions and coping responses on tinnitus adjustment, but only separate ly. The results from these studies are in line with the assumptions of the CSM. It has been found that negative illness representations, i.e. a strong illness identity, perceptions of serious consequences, and perceptions of low control, were associated with lower levels of ad justment to tinnitus [6,13 15]. With regard to coping, researchers proposed that especially cognitive processes play an important role in tinnitus adjustment [16]. Studies showed that coping strategies such as dwelling on tinnitus, catastrophizing about consequences, and masking the tinnitus were related to more psychological distress, whereas strategies such as acceptance, positive self talk, and engag ing in activities were related to less psychological distress [17 20]. To our knowledge, there are no studies that have examined the pos tulated mediating effect of coping responses on the relationship be tween illness representations and adjustment in patients with chronic tinnitus.

Therefore, the aim of the present study was to extend previous re search on tinnitus adjustment by jointly investigating the impact of illness representations and coping responses, i.e. positive and 
negative self instructions, on tinnitus related complaints based on the assumptions of the CSM.

\section{Method}

\section{Participants and procedure}

Participants were recruited from the Tinnitus Center of the Uni versity of Regensburg, Germany. A total of 200 patients diagnosed with tinnitus were invited to participate. They were provided with in formation about the study and received a booklet containing the questionnaires. A total of 118 patients returned the completed book let (response rate: 59\%). The study was approved by the Ethical Com mittee of the University of Regensburg and informed consent was obtained from all participants.

\section{Measures}

\section{Illness Perceptions Questionnaire (IPQ R)}

Participants' cognitive and emotional representations of tinnitus were assessed by a modified German version of the Illness Percep tions Questionnaire Revised [21,6].

The first part measures illness identity that refers to symptoms per ceived to be part of the illness. For 15 symptoms, participants indicat ed whether they have experienced the symptom in the course of their tinnitus, and if yes, whether they believe the symptom to be specifi cally related to their tinnitus.

The second part contains 7 subscales assessing chronic timeline (5 items), cyclical timeline (4 items), consequences (5 items), personal control (4 items), treatment control (4 items), coherence (5 items), and emotional representations (5 items). Responses were given on a 5 point rating scale. Cronbach's alpha ranged between .65 and .91 , ex cept for cyclical timeline $(\alpha=.51)$ which was therefore excluded from further analyses.

The third part measures causal attributions. Participants rated 21 potential causes of their tinnitus. A principal component analysis with VARIMAX rotation yielded two factors that can be labeled as in ternal causes ( 7 items, $\alpha=.86$ ) and external causes ( 5 items, $\alpha=.62$ ).

\section{Tinnitus Related Self Statements Scale (TRSS)}

The Tinnitus Related Self Statement Scale [22] was used to assess situation specific cognitions during the occurrence of tinnitus. The TRSS consists of two subscales capturing negative self instructions ( 9 items, $\alpha=.86$ ) and positive self instructions ( 9 items, $\alpha=.80$ ).

\section{Tinnitus Questionnaire (TQ)}

The German version of the Tinnitus Questionnaire [23] was used to assess tinnitus related complaints. The TQ contains 5 subscales re lating to psychological distress, intrusiveness, auditory perception difficulties, sleep disturbance, and somatic complaints. A total score that includes 42 items was calculated $(\alpha=0.95)$.

\section{Statistics}

Data were analyzed following the procedure described by Evans and Norman [11]. A regression analysis quantified the extent to which illness representations and self instructions explained vari ance in tinnitus related complaints. Illness representations were en tered in the first step and self instructions were entered in the second step. Subsequent multiple mediation analyses [24] were con ducted when the effect of an illness representation dimension in step 1 was substantially reduced (by at least 0.5 points) after the addition of self instructions in step 2 in order to test whether self instructions mediated the effect of the illness representation dimension on tinnitus related complaints.

\section{Results}

Sample characteristics

The sample consisted of 27 women and 91 men with a mean age of 55.64 years $(S D=11.31)$. The majority of participants have received some kind of tinnitus treatment in the past (94.8\%). Most participants were employed $(57 \%)$ or retired (36\%), while only a few received illness benefits $(2 \%)$ or were unemployed/homemakers (3\%). The mean sum score for the overall subjective severity of tinnitus (TQ-score $M=40.49, S D=17.87$ ) was below the cut-off point for decompensated tinnitus, although 28 (23.7\%) participants reported severe distress (TQ-score: 47-59) and 16 (13.6\%) participants reported very severe distress (TQ-score: 60-84).

Explained variance in tinnitus-related complaints and mediating effects of self-instructions

The illness representations entered in the first step of the regression explained 57\% of the variance in tinnitus-related complaints (see Table 1). The dimensions identity, consequences, coherence, and emotional representations showed significant associations with the outcome. The addition of the self-instructions in the second step of the regression produced a significant increment by $5 \%$ in the amount of explained variance. Negative self-instructions were significantly associated with the outcome.

As the effects of consequences and emotional representations were substantially reduced when the self-instructions were added to the regression, two multiple mediation analyses were conducted. Both analyses revealed a significant mediation effect of negative self-instructions (see Table 2), indicating that the use of negative selfinstructions partially mediated the effect of consequences and fully mediated the effect of emotional representations on tinnitus-related complaints.

\section{Discussion}

The present findings confirm the assumption that tinnitus adjust ment is strongly influenced by psychological factors. Thus, the way people think and feel about their tinnitus and the way they cope with their tinnitus are substantially related to their adjustment to tin nitus. Among the illness representations, the dimensions identity, consequences, coherence, and emotional representations are of spe cial importance which is in line with previous research on tinnitus sufferers [9] and other patients [10]. Attributing many symptoms to tinnitus, the perception of serious consequences, a low sense of un derstanding of the tinnitus, and strong emotional representations are directly and, in part, indirectly via negative self instructions associated with elevated levels of tinnitus related complaints. With regard to coping, negative self instructions have an unfavorable effect on tinnitus adjustment which concurs with previous studies examin ing the effectiveness of coping strategies in tinnitus patients $[17,18]$. Positive self instructions were not independently associated with bet ter adjustment. Additionally, negative self instructions mediated at

Table 1

Results of the regression analysis

\begin{tabular}{lll}
\hline \multicolumn{1}{c}{ Independent variables } & $\beta$ step 1 & $\beta$ step 2 \\
\hline Step 1: adj. $R^{2}=.58, F(7,103)=22.97^{* * *}$ & $.23^{* *}$ & $.20^{* *}$ \\
Identity & .09 & .07 \\
Chronic timeline & $.33^{* * *}$ & $.27^{* *}$ \\
Consequences $^{\mathrm{a}}$ & $.18^{*}$ & $.15^{*}$ \\
Coherence $_{\text {Emotional representations }}^{\mathrm{a}}$ & $.28^{* *}$ & .14 \\
Internal causes $_{\text {External causes }}$ & .01 & .04 \\
Step 2: $\Delta R^{2}=.05, F(2,101)=7.05^{* *} ;$ adj. $R^{2}=.63, F(9,101)=21.54^{* * *}$ & .03 \\
Negative self-instructions & & $.35^{* * *}$ \\
Positive self-instructions & & .06
\end{tabular}

Note. Only those illness representation dimensions that correlated significantly with tinnitus-related complaints were entered into the regression analysis in order to keep the number of independent variables to a minimum.

a Mediation was subsequently tested (see Table 2).

*** $p<.001$

** $p<.01$.

$* p<.05$. 
Table 2

Results of the mediation analyses

\begin{tabular}{llll}
\hline Effect & $\beta$ & BCa 95\% CI & \\
\hline & & Lower & Upper \\
Independent variable: consequences & & & \\
IV - Mediator 1 & $.22^{*}$ & & \\
IV - Mediator 2 & $.25^{*}$ & & \\
Mediator 1 - DV (direct effect) & $.36^{* * *}$ & & \\
Mediator 2 - DV (direct effect) & .06 & & .189 \\
IV - DV (total effect) & $.33^{* * *}$ & & .011 \\
IV - DV (direct effect) & $.27^{* *}$ & & \\
Mediation mediator 1 & $.08^{\mathrm{a}}$ & .018 & \\
Mediation mediator 2 & $.02^{\mathrm{a}}$ & .077 & \\
Independent variable: emotional representations & & \\
IV - Mediator 1 & $.44^{* * *}$ & & .19 \\
IV - Mediator 2 & .19 & & $.36^{* * *}$ \\
Mediator 1 - DV (direct effect) & .06 & & .010 \\
Mediator 2 - DV (direct effect) & $.28^{* *}$ & & \\
IV - DV (total effect) & .14 & & .057 \\
IV - DV (direct effect) & $.16^{\mathrm{a}}$ & & \\
Mediation effect mediator 1 & $.01^{\mathrm{a}}$ & .064 & \\
Mediation effect mediator 2 &
\end{tabular}

Note. Mediation analyses were calculated with consequences/emotional representations as independent variable (IV), negative and positive self-instructions as mediators 1 and 2 , tinnitus-related complaints as dependent variable (DV), and the remaining dimensions of illness representations as covariates.

BCa $95 \% \mathrm{CI}=$ bias corrected and accelerated $95 \%$ confidence interval; 5000 bootstrap samples.

a The mediation effects were estimated by bootstrap analyses [25]

$* * * \quad p<.001$.

$* * \quad p<.01$.

$* p<.05$.

least partially the unfavorable impact of consequences and emotion al representations on tinnitus adjustment. Thus, strong emotional representations and the perception of serious consequences promote the use of negative self instructions which, in turn, hinder adjust ment to tinnitus.

These findings are of practical interest as they provide an indica tion of which aspects should mainly be targeted in psychological and psychotherapeutic interventions for tinnitus, namely, negative illness representations and catastrophizing. Patients with chronic tin nitus may benefit from information provision on and discussions about the symptoms, timeline, possible treatments, and likely conse quences of tinnitus as this might foster their sense of understanding of tinnitus. It might also lead to an increase of patients' perceptions of control which, in turn, might minimize the emotional impact of tin nitus and reduce the perceived severity of consequences. Interven tions developed to modify illness representations in other patient groups could generate the desired changes [26,27]. Additionally, pa tients should be dissuaded from negative self instructions and instructed on how to deal with the tinnitus and how to accommodate to its consequences. Previous research identified acceptance of the tinnitus and engaging in activities as effective ways of coping with tinnitus $[19,20]$. The suggestions derived from our findings are in line with cognitive behavioral therapy (CBT) which has been found to have positive effects on qualitative aspects of tinnitus and the man agement of tinnitus. CBT for tinnitus usually combines the provision of information, the modification of negative thinking, and the en hancement of effective coping $[28,29]$. The present findings could help to specify the areas of negative cognitions and indicate that es pecially negative illness perceptions and catastrophizing should be targeted in psychological tinnitus treatment. Furthermore, our find ings could also be of use in the context of tinnitus counseling as the manner in which information about tinnitus and its treatment is com municated to patients influences their illness representations and their use of coping strategies.

Some limitations need to be acknowledged. Due to the cross sectional design, the direction of causality in the associations between the constructs could not be determined. Longitudinal studies are needed to clarify this issue. Additionally, longitudinal studies could investigate the dynamic processes specified in the CSM [30]. From the various possible ways of coping with tinnitus, this study focused on two cognitive coping responses. Further research would profit from including more diverse coping strategies to get a broader insight in the interplay between illness representations, coping responses, and tinnitus related complaints.

\section{Role of funding source}

Funding was provided by the Committee for Research Funding (AFF) of the University of Konstanz, Germany to Natallia Kalkouskaya and Manja Vollmann. The AFF had no further role in study design; in collection, analysis and interpretation of the data; in the writing of the report; and in the decision to submit the paper for publication.

\section{Conflict of interest}

The authors have no competing interests to report.

\section{References}

[1] Lockwood AH, Salvi RJ, Burkard RF. Tinnitus. N Engl J Med 2002;347:904-10.

[2] Sanchez L. The epidemiology of tinnitus. Audiol Med 2004;2:8-17.

[3] Andersson G, Vretblad P, Larsen HC, Lyttkens L. Longitudinal follow-up of tinnitus complaints. Arch Otolaryngol Head Neck Surg 2001;127:175-9.

[4] Sanchez L, Stephens SDG. Perceived problems of tinnitus clinic clients at long-term follow up. J Audiol Med 2000;9:94-103.

[5] Langguth B, Landgrebe M, Kleinjung T, Sand GP, Hajak G. Tinnitus and depression. World J Biol Psychiatry 2011;12:489-500.

[6] Reynolds P, Gardner D, Lee R. Tinnitus and psychological morbidity: a cross-sectional study to investigate psychological morbidity in tinnitus patients and its relationship with severity of symptoms and illness perceptions. Clin Otolaryngol Allied Sci 2004;29:628-34.

[7] Andersson G, Baguley DM, McKenna L, McFerran DJ. Tinnitus: a multidisciplinary approach. London: Whurr; 2005.

[8] Ward LM, Baumann M. Measuring tinnitus loudness using constrained psychophysical scaling. Am J Audiol 2009;18:119-28.

[9] Leventhal H, Meyer D, Nerenz D. The common sense model of illness danger. In: Rachman S, editor. Medical psychology. New York: Pergamon; 1980. p. 7-30.

[10] Hagger MS, Orbell S. A meta-analytic review of the common-sense model of illness representations. Psychol Health 2003:18:141-84.

[11] Evans D, Norman P. Illness representations, coping and psychological adjustment to Parkinson's disease. Psychol Health 2009;24:1181-96.

[12] Förster C, Taubert S. Subjektive Krankheitstheorien und Selbstregulation von Tumorpatienten. Z Med Psychol 2006;15:117-27.

[13] Budd RJ, Pugh R. The relationship between locus of control, tinnitus severity, and emotional distress in a group of tinnitus sufferers. J Psychosom Res 1995;39: 1015-8.

[14] Sirois FM, Davis CG, Morgan MS. "Learning to live with what you can't rise above": control beliefs, symptom control, and adjustment to tinnitus. Health Psychol 2006;25:119-23.

[15] von Osterhausen K, Kessler B, D'Amelio R, Delb W. Adaptivität und Maladaptivität der Krankheitsverarbeitung bei Patienten mit subjektivem chronischen Tinnitus. Verhaltenstherapie Verhaltensmedizin 2001;22:39-54.

[16] Andersson G, McKenna L. The role of cognition in tinnitus. Acta Otolaryngol 2006;126:39-43.

[17] Budd RJ, Pugh R. The relationship between coping style, tinnitus severity and emotional distress in a group of tinnitus sufferers. Br J Health Psychol 1996;1: 219-29.

[18] Budd RJ, Pugh R. Tinnitus coping style and its relationship to tinnitus severity and emotional distress. J Psychosom Res 1996;41:327-35.

[19] Davis CG, Morgan MS. Finding meaning, perceiving growth, and acceptance of tinnitus. Rehabil Psychol 2008;53:128-38.

[20] Westin V, Hayes SC, Andersson G. Is it the sound or your relationship to it? The role of acceptance in predicting tinnitus impact. Behav Res Ther 2008;46: 1259-65.

[21] Gaab J, Latanzia-Bunschoten S, Sprott H, Ehlert U. IPQ. Illness Perception Questionnaire (Deutsche version). In: Bengel J, Wirtz M, Zwingmann C, editors. Diagnostische Verfahren in der Rehabilitation. Göttingen: Hogrefe; 2008. p. 109-11.

[22] Weise C, Heinecke K, Rief W. Biofeedback-based behavioral treatment for chronic tinnitus: results of a randomized controlled trial. J Consult Clin Psychol 2008;76: 1046-57.

[23] Goebel G, Hiller W. Tinnitus-Fragebogen. Göttingen: Hogrefe; 1998.

[24] Preacher KJ, Hayes AF. Asymptotic and resampling strategies for assessing and comparing indirect effects in multiple mediator models. Behav Res Methods 2008;40:879-91.

[25] Shrout PE, Bolger N. Mediation in experimental and nonexperimental studies: new procedures and recommendations. Psychol Methods 2002;7:422-45. 
[26] Broadbent E, Ellis CJ, Thomas J, Gamble G, Petrie KJ. Further development of an illness perception intervention for myocardial infarction patients: a randomized controlled trial. J Psychosom Res 2009;67:17-23.

[27] Glattacker M, Heyduck K, Meffert C. Illness beliefs, treatment beliefs and information needs as starting points for patient information - evaluation of an intervention for patients with chronic back pain. Patient Educ Couns 2012;86:378-89.

[28] Martinez-Devesa P, Waddell A, Perera R, Theodoulou M. Cognitive behavioural therapy for tinnitus. Cochrane Database Syst Rev 2007(1) [Art. No.: CD005233].
[29] Zachriat C, Kröner-Herwig B. Treating chronic tinnitus: comparison of cognitivebehavioural and habituation-based treatments. Cogn Behav Ther 2004;33: 187-98.

[30] Leventhal H, Benyamini Y, Brownlee S, Diefenbach M, Leventhal EA, Patrick-Miller L, et al. Illness representations: theoretical foundations. In: Petrie KJ, Weinman JA editors. Perceptions of health and illness: current research and applications. Harwood Academic Publishers: Amsterdam, The Netherlands; 1997. p. 19-45. 